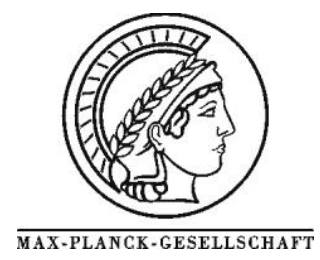

\title{
Buchbesprechung: In-Situ Spectroscopy in Heterogeneous Catalysis Herausgegeben von James F. Haw
}

\author{
F. C. Jentoft
}

Fritz-Haber-Institut of the Max Planck Society Faradayweg 4-6, 14195 Berlin

Die Notwendigkeit, Erkenntnisse darüber zu gewinnen, wie heterogene Katalysatoren im Detail ,arbeiten“, hat die Entwicklung von In-situ-Methoden in den letzten Jahrzehnten enorm vorangetrieben. Ein zusammenfassender Überblick über dieses Forschungsgebiet ist deshalb angebracht. Das von James Haw herausgebrachte Buch ist eine Sammlung von Beiträgen ausgewiesener Experten über ausgewählte Insitu- Verfahren (nicht alle sind spektroskopische). Das Werk zielt darauf ab, ,young scientists with training in diverse areas" in das Forschungsgebiet Heterogene Katalyse einzuführen.

In der Einleitung spricht der Herausgeber die derzeitigen Grenzen - „pressure gap“, „,materials gap“, ,,spectator species" - und den Einfluss der Theorie an und stellt den Bezug zu den einzelnen Kapiteln her. Auch versucht Haw, den Begriff ,in situ“ zu definieren. Dieser Abschnitt trägt die Überschrift „,compromises“, und demgemäß lässt er nicht nur eine saubere Definition zu, sondern auch einen pragmatischen Ansatz, in dem die Bezeichnung ,in situ“ auf jede Untersuchung zutrifft, ,that teaches us about the chemistry... under reaction conditions".

Alle Beiträge sind gleich aufgebaut: Nach einer Einleitung in das jeweilige Verfahren werden einige Beispiele vorgestellt. Eine Frage, die sich stellt, ist, inwieweit ein derartiges Buch auf die grundlegenden Prinzipien der Methoden eingehen soll. Dieses Problem ist recht gut gelöst, indem nur die ausgefalleneren Verfahren, die nicht in allgemeinen Lehrbüchern zu finden sind, ausführlich beschrieben werden. Ein weiterer Vorteil des Buchs ist, dass die Beiträge hinsichtlich inhaltlicher Tiefe und vorauszusetzender Vorkenntnisse ein einheitliches Niveau haben.Wer sich allerdings über technische Details informieren will, muss auf die angegebene Literatur zurückgreifen.

Abbildungen, die experimentelle Aufbauten oder Daten zeigen, sind reichlich vorhanden und tragen zur hohen Qualität des Buchs bei. Diese graphischen Darstellungen sind in der Regel informativ, übersichtlich und gut erläutert. Die ausgiebige Verwendung von Untertiteln in den Kapiteln ist sehr hilfreich, da diese Abschnittsüberschriften im Inhaltsverzeichnis sorgfältig aufgelistet sind und somit die Strukturierung der Kapitel gut widerspiegeln. Das Inhaltsverzeichnis ist für den Leser zum Auffinden bestimmter Themen nützlicher als das Sachwortverzeichnis, das viele Fehler und Wiederholungen aufweist.

Das 1. Kapitel beschäftigt sich mit Oberflächenphysik, wobei zunächst die „sum frequency generation“(SFG)Methode (Schwingungsspektroskopie durch Mischen infraroter und sichtbarer Laserstrahlung) im Mittelpunkt steht und das Potenzial eines echten In-situ- Experiments eindrucksvoll aufgezeigt wird: Die katalytischen und spektroskopischen Daten der untersuchten Oxidation von CO am Pt-Kontakt stehen tatsächlich in direktem Zusammenhang. Der zweite Teil dieses Kapitels ist der Rastertunnelmikroskopie gewidmet und befasst sich ausschlie ${ }^{\circ}$ lich mit adsorbierten Spezies und nicht direkt mit Katalyse. Es wird aber ein wichtiger Punkt aufgebracht, nämlich experimentell herbeigeführte Prozesse (,tip-induced catalysis“).

Das vom Herausgeber verfasste Kapitel gibt einen guten «berblick über die Möglichkeiten zum Einsatz der NMR-Spektroskopie in In-situ-Untersuchungen der Katalyse. Als geeignetste Kernart hat sich dabei bisher ${ }^{13} \mathrm{C}$ erwiesen, und somit liegt der Schwerpunkt auf ${ }^{13} \mathrm{C}-\mathrm{NMR}$ Studien, die Informationen über die Oberflächenspezies und die Produkte liefern, allerdings nicht über Veränderungen des Katalysators selbst. Die Beispiele zeigen, wie mühselig und umständlich diese Methode ist. Die Ausführungen sind sehr ehrlich in Bezug auf die Grenzen der Methode: „(NMR) cannot work alone”. Auf diese Aussage folgt ein Beispiel, in dem sich NMR-Spektroskopie und eine weitere Methode sinnvoll ergänzen.

Im folgenden Kapitel zur Theorie werden die Prinzipien der Rechenmethoden zusammengefasst, was zu einem Streifzug durch die Akronyme der Theoretischen Chemie 
gerät. Eine Stärke dieses Beitrags ist die gewaltige Zahl der angegebenen Literaturverweise. Die Beispiele stammen alle aus dem Bereich der Säure-Base-Katalyse, beruhen auf Clustermodellen und bieten einen Vergleich der berechneten Energien (Aktivierung, Adsorption) und chemischen Verschiebungen (NMR) mit experimentellen Ergebnissen.

Das Kapitel über Raman-Spektroskopie ist geprägt durch die Forschungs- arbeiten des Autors (UV-Raman). Obwohl die Herausforderungen einer Insitu-RamanMethode klar dargelegt werden, sind die angeführten Beispiele (eines betrifft nicht die Katalyse) nicht dazu geeignet, die Vorzüge eines In-situ- Experiments zu demonstrieren: Über die Beschaffenheit von Koks auf einem Zeolithen und die Zersetzung von Schmiermitteln beim Zerreiben mit verschiedenen Werkzeugen wird berichtet.

Das Kapitel über Röntgenabsorptionsspektroskopie (XAS) betont methodische Aspekte. Einige neuere Entwicklungen von Zellen und die wichtigen schnellen sowie dispersiven Verfahren werden nicht erwähnt. Die Kompliziertheit der Datenanalyse wird nur oberflächlich erörtert und die Beispiele werden äußerst gedrängt dargestellt. Ein nützlicher Hinweis auf die Anwendung von In-situTechniken bei der Herstellung von Katalysatoren wird gegeben.

Im achten Kapitel wird dem Leser eine umfassende Einführung in die Anwendungen der Positronenemission in In-situ-Experimenten geboten. Untersuchungen axialer Konzentrationsprofile in Durchflussreaktoren werden ebenfalls diskutiert. Dieses längste Kapitel des Buchs enthält auch eine ausführliche Beschreibung der mathematischen
Modellierung zur Datenberechnung, z.B. von Konzentrationsprofilen in einem Pulverbett.

Die Kapitel über IR-spektroskopische Methoden und „temporal analysis of products“ (TAP) sind sehr informativ und interessant. Eine Reihe von IRUntersuchungsverfahren, darunter wiederum SFG-Methoden, einschließlich Zellenkonstruktionen, werden besprochen. Die Beispiele aus einem breit gefächerten Themenbereich zeigen die Stärken und Schwächen der IR-Methoden. Der Beitrag über TAP beeindruckt durch seinen klaren Aufbau und Informationsgehalt; die Experimente zur Analyse der Daten werden unter allen Aspekten beleuchtet.

Warum die UV/Vis-, die paramagnetische Elektronenresonanz-Spektroskopie und wichtige Methoden zur Oberflächenuntersuchung wie die Röntgen- Photoelektronenspektroskopie, die langsam in interessante Druckbereiche vordringen, nicht behandelt werden, ist ebenso unverständlich wie die Tatsache, dass auf mikroskopische Verfahren überhaupt nicht eingegangen wird; nicht einmal in der Einleitung. Ein Kapitel, in dem alle Angaben über die Untersuchungsmethoden wie erreichbare Temperaturund Druckbereiche, Empfindlichkeiten, Zeitauflösung usw. in Form von Tabellen zusammengefasst sind, hätte dem Leser einen kritischen Vergleich der Verfahren ermöglicht und das Buch gegenüber Übersichtsartikeln über einzelne Methoden hervorgehoben. Ein solches Kapitel fehlt leider, aber dennoch bietet das Buch interessanten Stoff für den Forscher, der sich mehr oder weniger mit Katalyse beschäftigt, und für den Spezialisten, der seine Kenntnisse erweitern will. 